\title{
Preliminary investigation of centrifugal pump for fire engine
}

\author{
Václav Dvořák ${ }^{1, *}$, Miloš Müller ${ }^{1}$ \\ ${ }^{1}$ Technical University of Liberec, Studentska 2, 46007, Liberec, Czech Republic
}

\begin{abstract}
This preliminary work deals with analytical, numerical and experimental investigation of centrifugal pump for fire engine. The pump was investigated experimentally and power curves as dependence of head on flow rate were carried out. Numerical research focused on finding of setup of numerical solver. All curves were compared, analysed and problematic places were identified. It was found that for very low backpressure, i.e. head coefficient, cavitation occurs around the propeller wheel and reduces maximum flow coefficient significantly. After modifying the wheel by sharpening leading edges of blades, or when rotation speed was increased, cavitation got even worse. Another issue is related with stator blades. It seems that stator blades work perfectly only in short range of regimes. Stator blades represent another resistance for low back pressure, while for higher back pressure, stator blades fail to decelerate the flow and increase static pressure. Several modification were suggested to increase performance of the fire engine.
\end{abstract}

\section{Introduction}

Centrifugal pump is a roto-dynamic device that uses a rotating impeller to increase the pressure of the pumped liquid. The liquid enters the pump along the axis of rotation and is accelerated within the impeller. The liquid leaves the impeller radially, entering a diffuser or a volute chamber. Centrifugal pumps are most common pump types used in power engineering, agriculture, industry and fire protection. The parameters describing the pump operation include Water Horsepower (WHP power delivered to the fluid), Brake Horse Power (BHP - the power required to drive the pump) and Net Positive Suction Head (NPSH - head required at the pump inlet to keep the liquid from cavitation). The ratio between the Water Horsepower and the Brake Horsepower defines the pump efficiency [1].

Cavitation is a term closely connected to the centrifugal pump operation. This represents an occurrence of vapour and gas phase within the body of liquid causing the decrease in the pump head [2]. Pump operation in cavitation regime can lead to the pump damage. Cavitation inception in centrifugal pumps can be characterised by dimensionless Cavitation number relating the pressure/inertial forces [1].

The presented paper focuses on the design and optimisation of fire pumps. In contrast to common industrial pumps, fire pumps operates in different regimes, which results in different design objectives [3]. Fire pumps are designed with very low NPSH value to avoid cavitation causing decreasing in the flow and head.

Stable and continuous decreasing flow-head curves are required to achieve a stable outlet pressure. Maximum flow is mainly limited by the cavitation occurrence and the flow capacity reserve. Most of fire pumps have constant rising BHP with the flow rate. The shapes of the fire pump characteristics are usually defined by national standards as NFPA 20 (Standard for the Installation of Stationary Pumps for Fire Protection) [3].

An effective pump design requires comprehensive analysis including both experimental and numerical methods. In recent years development in Computational Fluid Dynamics (CFD) enables detailed modelling of the roto-dynamic machines including multiphase flow and cavitation. Currently the last development is focused on the two-phase flow (cavitation) including the Fluid Structure Interaction FSI [4].

Three different methods are used to simulate the rotating turbomachinery flow: Multiple Reference Frame (MRF), Mixing Plane and Sliding Mesh. In the MRF method the stator is solved in fixed reference frame and the impeller is solved in rotating reference frame including dynamic fluid forces [5], [6]. As the MRF and Sliding Plane models are steady state approximations for the transient rotor-stator interaction, Sliding Mesh is desired. The approaches mentioned above enables testing of pump characteristic for different geometrical configurations before the pump is manufactured.

Barrio et al. [7] solved full 3D-URANS equations using the sliding mesh technique to estimate radial load on impeller of a centrifugal pump when operating at offdesign conditions.

Jafarzadeh et al. [8] applied standard k- $\varepsilon, \mathrm{RNG}$ and RSM turbulence models to simulate the flow within a low-specific-speed high-speed centrifugal pump. The effect of blade number for 5 and 7 blades on the pump characteristics was tested. The results showed higher head coefficient for impeller with 7 blades.

\footnotetext{
* Corresponding author: vaclav.dvorak@tul.cz
} 
Liu et al. [9] tested an influence of empirical coefficients of the Zwart-Gerber-Belamri cavitation model on prediction of cavitating flow in centrifugal pump.

Gonzalez et al. [10] presented the capability of a numerical simulation to capture the dynamic of the unsteady flow inside the centrifugal pump due to the Impeler-Volute interaction. The unsteady viscous Navier-Stokes equations in the combination with the sliding mesh technique are used to show flow and pressure fluctuations due to impeller-volute interaction.

Medvitz et al. [11] applied multiphase method to analyse centrifugal pump performance under developed cavitation conditions. Two phase RANS were used within the simulation. The rapid drop in the head coefficient at low cavitation numbers was observed. Characteristic trends associated with the blade cavitation were observed.

Nohmi et al. [12] tested two types of cavitation CFD codes. A locally homogeneous model of compressible gas-liquid two phase medium and Constant Enthalpy Vaporization (CEV) models were tested. The steep head drop was well captured by both models in the cavitation occurrence. The models enables the prediction of NPSH at the cavitation breakdown point.

Jiang et al. [13] used numerical simulation based on the Reynolds-averaged Navier-Stokes (RANS) combined with the SST $\mathrm{k}-\omega$ turbulence models to investigate the influence of the clocking effect on the unsteady pressure fluctuations and radial forces in the centrifugal pump with vaned diffuser.

Although plenty of articles regarding the CFD aided centrifugal pump design have been published, only a few papers have been focused on the CFD aided fire pump design. The difference in the common centrifugal pump and fire centrifugal pump design results from the different utilization conditions, which were noted in the previous text. This paper utilizes three different approaches for obtaining the fire pump performance parameters, which enables to verify their relevance.

\section{Methods}

\subsection{Analytical method}

Centrifugal pumps are not usually provided with guiding vanes in front of the propeller wheel. Therefore, total pressure increase, which obtains the fluid, i.e. Euler equation is given by

$$
\Delta p=\rho u_{2} c_{2 u},
$$

where $u_{2}$ is outlet blade velocity and $c_{2 u}$ is component of outlet absolute velocity $c_{2}$ in the direction of blade velocity.

Above equation predicts too high power compare to experiments. To add some empirical experience, we used efficiency of diffuser $\eta_{d i f}$ to consider its imperfection to transform kinetic energy into pressure energy. The static pressure obtained by fluid is given by equation

$$
\Delta p_{\text {stat }}=\Delta p-\rho \frac{c_{2}^{2}}{2}\left(1-\eta_{\text {dif }}\right) .
$$

To compare results obtained at different conditions, two dimensionless numbers are used. The flow coefficient $\varphi$ is a dimensionless quantity used to describe the volume flow rate. It is also referred to as the volume or volume flow coefficient and characterises the flow rate. It is defined as

$$
\varphi=\frac{Q}{\pi d_{2} b_{2} u_{2}},
$$

where $Q\left(\mathrm{~m}^{3} / \mathrm{s}\right)$ is volumetric flow rate, $d_{2}(\mathrm{~m})$ is outlet diameter of the pump wheel and $b_{2}(\mathrm{~m})$ is outlet width.

The head coefficient $\psi$ is a characteristic coefficient derived from the corresponding physical quantity according to the affinity laws and used to characterise the operating behaviour. It characterises the head of the pump

$$
\psi=\frac{\Delta p}{\varrho \frac{u_{2}^{2}}{2}} .
$$

\subsection{Numerical method}

Numerical investigation was done in Fluent software. We used two different methods to simulate rotations of the wheel: Moving reference frame method with steady solver and dynamic mesh with transient solver. While using moving reference frame method, a rotational relative velocity is specified for volumes of fluid inside the mesh of the wheel instead of real rotation which is simulated while using dynamic mesh.

The computational domain is visible in Figure 1 and Figure 2. The fire engine has got one inlet for water suction and two outflow which allow connection of two hoses, see Figure 1. The impeller, which is in Figure 2, has six backward curved blades with blunt leading edges. 


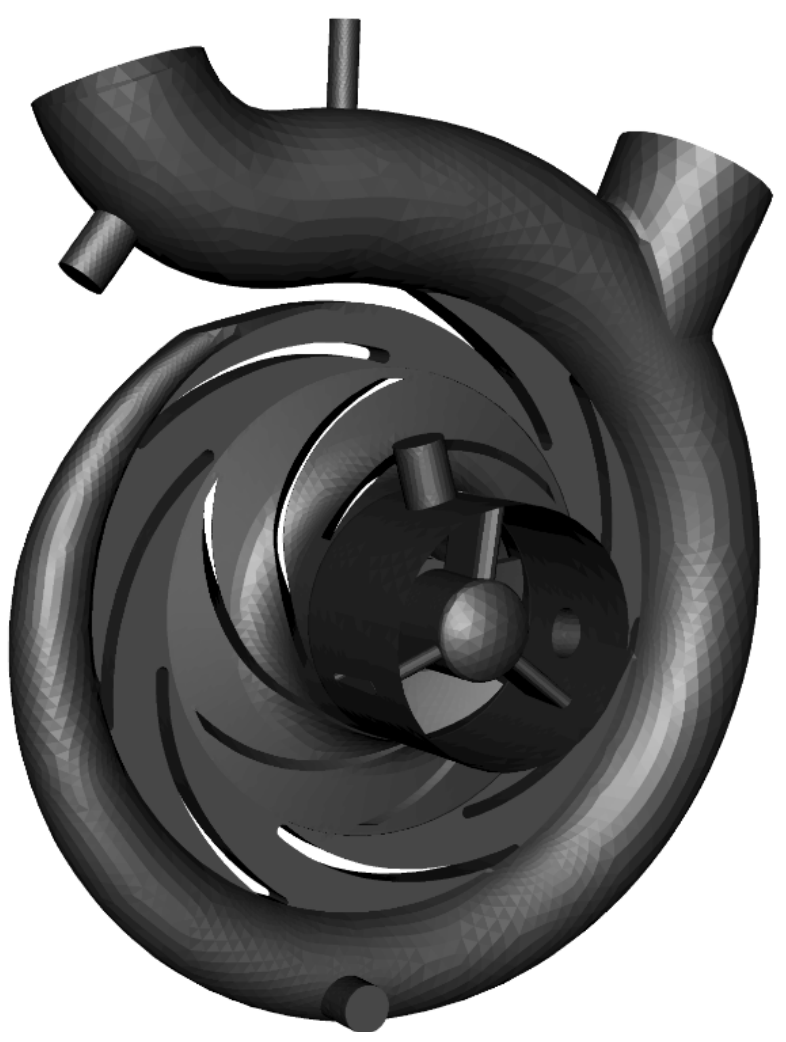

Fig. 1. Numerical model of fire engine - inlet, casing and outlets.

Seven stator blades are placed between wheel outlet and inlet into the diffuser.

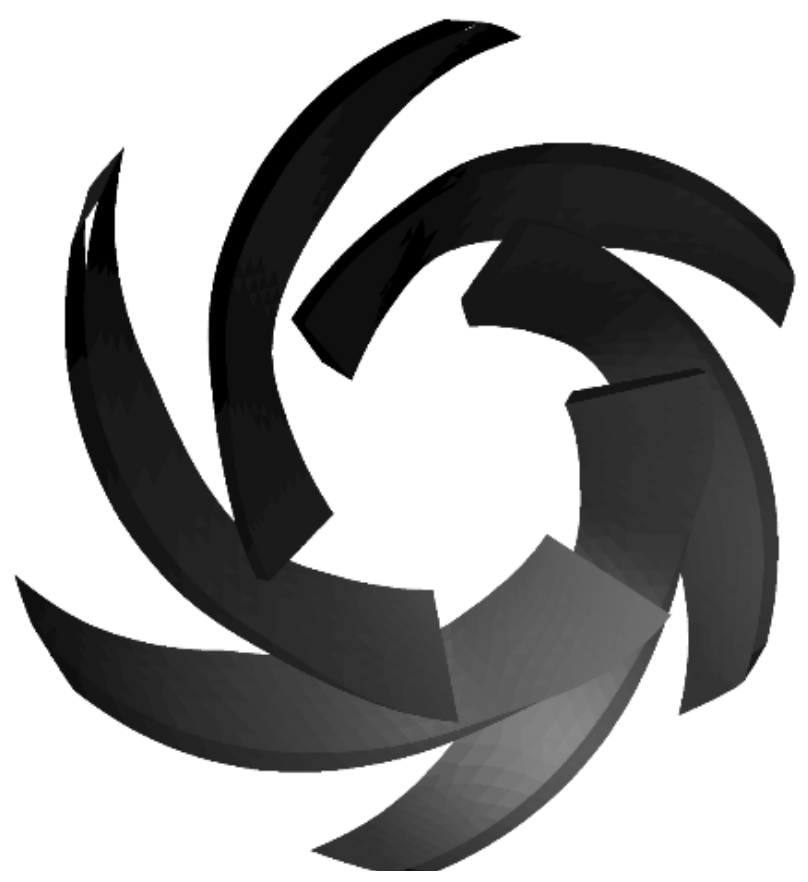

Fig. 2. Numerical model of fire engine - rotor blades.

To get closer to conditions that were during measurement, we used so-called inlet-vent as inlet boundary conditions which allows to specify pressure jump caused friction and local losses during suction the fluid. Similarly, outlet-vent was used on the outlets to simulate friction in connected hoses.
We used pressure-based solver, which is suitable for incompressible fluids, and k- $\omega$ SST model [14] to solve turbulent flow.

This paper utilizes experimental measurement, CFD modelling and analytical approaches for obtaining the fire pump performance parameters. The analytical approach offers an inexpensive view into the problem without any information about the space and time distribution of the flow. The CFD modelling represents a robust tool for the mapping of the complex flow with high spatial and temporal resolution. In principal all important physical phenomena can be included, but the results are strongly influenced by the selection of proper turbulence and cavitation models. The experimental model can be used for the validation of the CFD and analytical approaches, however the compatibility of the boundary conditions and the correct experimental results interpretation have to be ensured.

\section{Results}

\subsection{Numerical investigation}

Several numerical solver setups were tested during the numerical investigation. The influence of the wall roughness on the pump performance curves was also tested. From the real pump inspection it was found that the height of surfaces roughness, which are not treated after casting, is between 0.2 and $0.5(\mathrm{~mm})$. Comparison of performance curves obtained for these two values and smooth walls is in Figure 3.

We can see significant reduction in obtained flow rate between smooth walls and roughness height equal to $0.2(\mathrm{~mm})$, but only negligible change between height 0.2 and $0.5(\mathrm{~mm})$ for low head and high flow rate. However, the difference between roughness 0.2 and $0.5(\mathrm{~mm})$ is still significant for higher head and low flow rates.

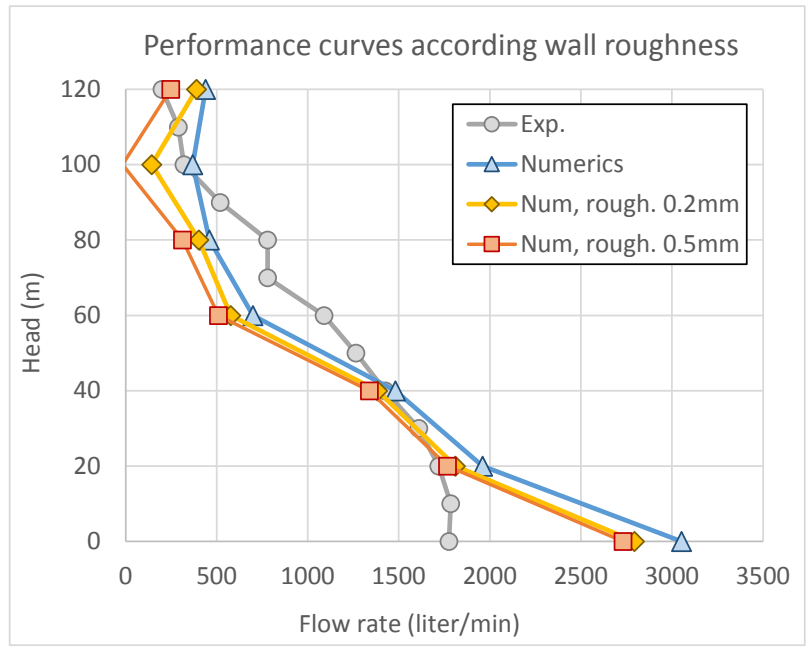

Fig. 3. Comparison of performance curves for various roughness height of walls.

As we can see in Figure 3, there is great discrepancy between computed and measured maximal volumetric flow rates. Figure 4 shows area weighted static pressures 
evaluated on propeller blades. This averaged static pressure was far below zero for highest flow rate which indicates that large cavitation region occurs in reality which causes significant reduction in maximal flow rate.

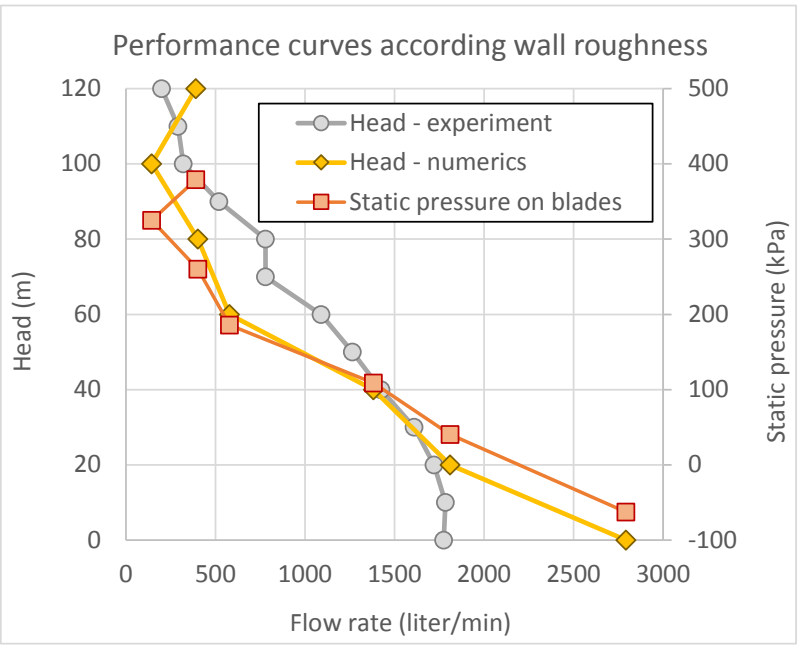

Fig. 4. Area averaged static pressure evaluated on rotor blades.

As we can further see in Figure 3, performance curves obtained for low flow rates are not smooth. It is caused by the unsteady behaviour of flow for high head regimes. As we can see in Figure 5, where instantaneous flow rates obtained during transient computation are drawn, the flow rates oscillate as rotor blades pass around stator blades. However, average flow is almost constant for low head which indicates stabile flow in the pump. For high head and low flow rates, the average flow rate does not remain constant which indicates flow separation and inappropriately working diffuser.

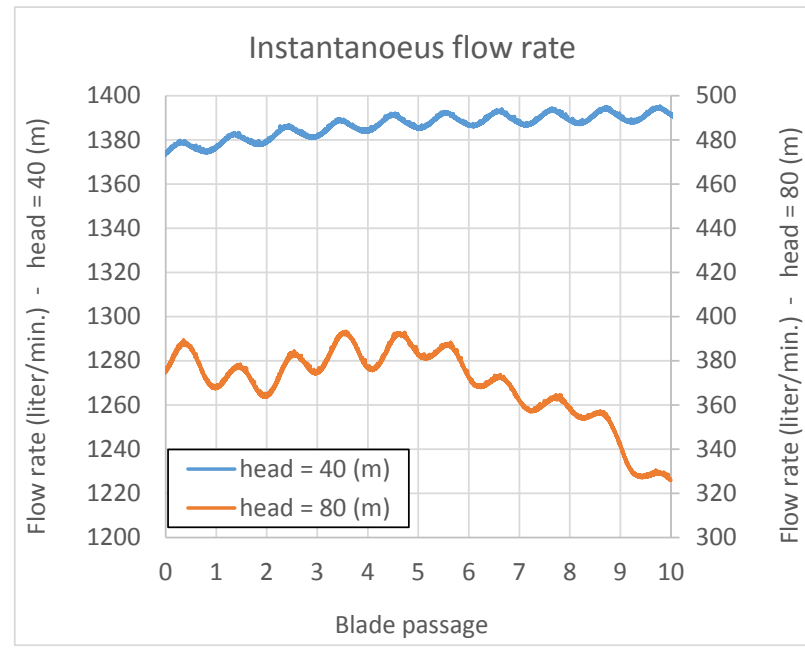

Fig. 5. Comparison of performance curves - dependence of head coefficient on flow coefficient.

This presumption is well confirmed by next Figures 6 and 7 , where path-lines coloured by velocity magnitude are drawn. While the flow in the pump wheel, in the diffuser and spiral casing is almost uniform for high flow rates and head $40(\mathrm{~m})$, see Figure 6, there is large reverse flow in the suction part of the pump and destroyed flow in the diffuser and spiral casing for low flow rates and head $80(\mathrm{~m})$, see Figure 7.

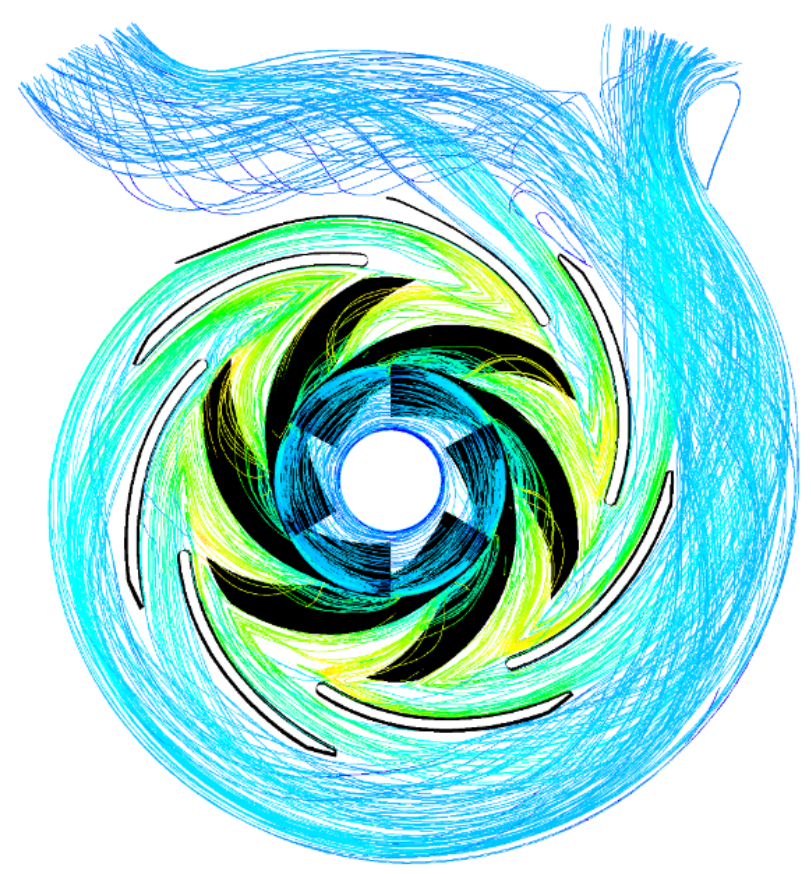

Fig. 6. Path-lines coloured by velocity magnitude for low head $40(\mathrm{~m})$, stator and rotor blades are visible.

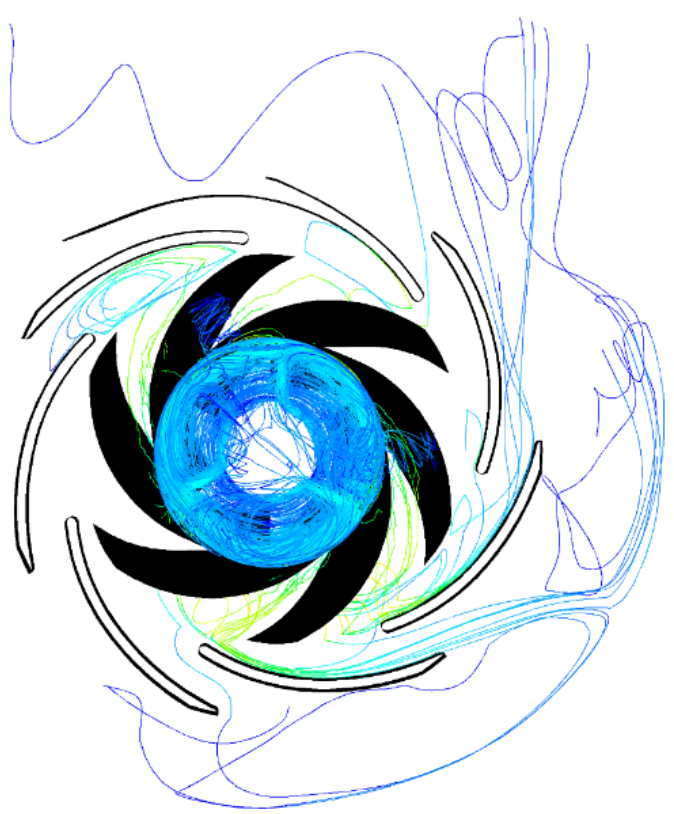

Fig. 7. Path lines coloured by velocity magnitude for low head $80(\mathrm{~m})$, stator and rotor blades are visible.

\subsection{Comparison with experiments}

Three experimentally measured performance curves, analytically calculated curves and a curve obtained from numerical simulation are compared in Figure 8. A significant difference in the curves is evident from the comparison. These distinctions are mainly caused by the changes in the speed of rotation during the measurement according to the drive engine load. 


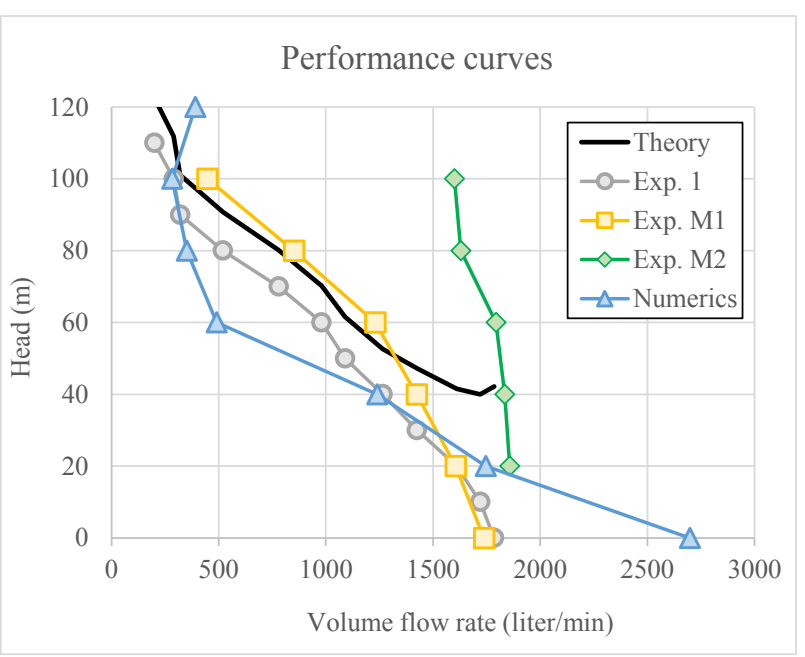

Fig. 8. Comparison of performance curves.

Dimensionless coordinates have to be used to compare results obtained under different conditions, see Figure 9. The first curve, Exp.1, was obtained for the original impeller. If a very low diffuser efficiency (about 0.16 ) of diffuser is used for calculation of head coefficient according equations (2) and (4), the curve Theory is very close to measured values for low flow coefficient $\varphi$ and high head coefficient $\psi$.

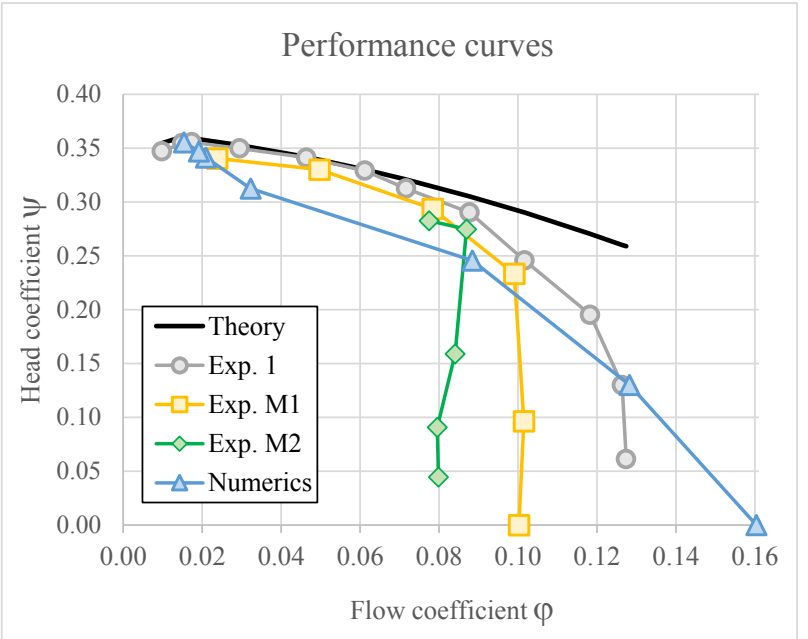

Fig. 9. Comparison of performance curves - dependence of head coefficient on flow coefficient.

From some value of the flow coefficient, $\varphi \cong 0.07$, the measured characteristic indicates faster head coefficient decrease than the theoretical one as the flow coefficient increases and reaches maximum value of 0.127 . This decrease can be explained by the presence of the cavitation.

The other characteristics Exp. M1 and Exp. M2 represents results of measurement for the modified impeller, which was produced by the leading edges machining. Created leading edge is sharp, which would improve the momentum transfer from the impeller to the liquid. As indicated from the Figure 9, the resulting power curve has lower level than the original impeller.
The maximum reached flow coefficient is lower than for the original geometry $(0.101)$. No improvement is visible after the modification. Probably the modification increased the cavitation occurrence, which is more evident from the characteristic Exp. M2 obtained for higher speed of rotation.

To characterize the possibility of the cavitation inception, cavitation number is evaluated as follows [1]

$$
\sigma=\frac{p_{1}-p_{v}}{\frac{1}{2} \cdot \rho \cdot u_{1}^{2}}
$$

where $p_{1}(\mathrm{~Pa})$ is the reference pressure (pressure at the pump inlet), where $p_{v}(\mathrm{~Pa})$ is the saturated vapour pressure at pump inlet temperature, $\rho\left(\mathrm{kg} / \mathrm{m}^{3}\right)$ is water density and $u_{1}(\mathrm{~m} / \mathrm{s})$ is a reference velocity (inlet tip speed velocity).

\section{Discussion}

Although there are large differences between the measured and calculated values of the flows achieved, the calculations qualitatively confirmed the measured data and helped to explain some of the measured phenomena.

First of all, it has been found that the measured pump has cavitation at low backpressures, and that the adjustment of the wheel - sharpening of the leading edges of the impeller blades, as well as the increase in speed - causes this situation to deteriorate.

It has also been shown that stator blades work well at high flow and low backpressure but have a rather adverse effect at low flow rates. The evaluated efficiency of the diffuser was about 0.16 .

Based on this finding, recommendations are made on what to look for when optimizing the fire pump. It is necessary to change the design of the impeller blades in order to avoid cavitation at high flow rate and further to modify the stator blades to improve the transformation of the kinetic energy of the water flow to the pressure energy.

Due to the persisting differences between the calculation and the measurement, it seems necessary to include in the calculation also the spaces in front of and behind the wheel, in which the fluid can escape through the gap between the rotor and the stator and thus help the flow in the pump at higher backpressures. Repeated calculations and measurements on the modified pump will further help to improve computational compliance with experimental research.

\section{Conclusions}

The flow in the impeller wheel and the diffuser of the fire pump has been investigated using analytical, numerical and experimental methods. The diffusor efficiency was considered for simple analytical method.

By comparing numerically and experimentally observed power curves, cavitation in the pump inlet was detected for high flow rates. This resulted in the pump engine relieve, increasing the engine speed and lowering 
the maximum available intake flow. The situation was further aggravated with the impeller modification by the sharpening of the leading edges of the impeller blades.

Both the measured and calculated curves showed that the flow in the pump diffuser is of design for higher backpressures. The flow separation, increased pulsations, and decreases in the achievable flow rate were indicated in these regimes.

In the real model a gap between the impeller output and the stator casing exists, which was neglected in current numerical model. Through the gap the liquid flows along the axis of rotation back to the impeller inlet and also to the cavity at the drive side. The flow from the cavity at the drive side can also be connected to an external chiller and returned back to the inlet. The future model will includes these additional cavities to obtain more realistic approach. As the cavitation occurrence is one of the limiting parameters of the fire pumps also the cavitation modelling is proposed.

\section{Acknowledgement}

This project was realized with financial support from the Technology Agency of the Czech Republic, grant no. TH03010378 "Development of a new range of fire pumps in extreme conditions".

\section{References}

1. A. M. White, Fluid mechanics, 764-771 (2009)

2. Ch. E. Brennen, Cavitation and Bubble Dynamics, 5-294 (1995)

3. B. Tchavdarov, D. Hardersen, A. Sdano, ASME. Fluids Engineering Division Summer Meeting, 1, 295-303 (2009)

4. Y. Tingyun, P. Ji, Y. Shouqi, O. M. Koranteng, W. Jiabin and W. Wenjie, J. Mech. Sci. Technol., 31(11), 5319-5328 (2017)

5. E. Dick, J. Vierendeels, S. Serbruyns, J. V. Voorde, Task Quarterly 5(4), 579-594 (2001)

6. J. Y. Luo, R. I. Issa, and A. D. Gosman, IChemE Symp. 136, 549-556 (1994)

7. R. Barrio, J. Fernández, E. Blanco, J. Parrondo, Eur J Mech B Fluids, 30 (3), 316-324 (2011)

8. B. Jafarzadeh, A. Hajari, M.M. Alishahi, M.H. Akbari, Appl. Math. Model., 35(1), 242-249 (2011)
9. H. Liu, Y. Wang, J. Wang, H. Huang, Int. J. Nav. Archit. Ocean Eng., 6(1), 119131 (2014)

10. J. Gonzalez, J. Fernandez, E. Blanco, C. Santolaria, J. Fluids Eng., 124(2), 348355 (2002)

11. R. B. Medvitz, R. F. Kunz, D. A. Boger, J. W. Lindau, A. M. Yocum, L. L. Pauley, ASME. J. Fluids Eng., 124(2), 377-383 (2002)

12. M. Nohmi, A. Goto, Y. Iga, T. Ikohagi, Proceedings of Fifth International Symposium on Cavitation - Osaka, 1, 17 (2003)

13.W. Jiang, G. Li, P. Liu, L. Fu, Int. Commun. Heat Mass, 71, 164-171 (2016)

14.F. R. Menter, AIAA Paper 93-2906, (1993) 